\title{
EFFICACY OF THE ACCENT METHOD OF VOICE THERAPY ON ACOUSTIC MEASUREMENTS, IN HYPERFUNCTIONAL DYSPHONIC PATIENTS
}

\author{
Nashwa Mahmoud Othman*, Mohmmed Ali Baraka, and Nahla Abdel-Aziz Rifaie
}

Phoniatrics Unit, Otorhinolaryngology Department, Faculty of Medicine, Ain Shams University, Cairo, Egypt

Corresponding author Nashwa Mahmoud Othman

Mobile: (+2) 01006732806

E.mail:

nashwa.m.othman@gmail.com

Received: 27/4/2021

Accepted: 26/5/2021

Online ISSN: 2735-3540

\begin{abstract}
Background: The accent method (AM) of voice therapy is one of the holistic approaches for behavior readjustment technique (BRAT). Anatomic physiologic explanation for the accent method is the rhythmic pairing of timing with respiration and phonation which may promote motor learning of new phonatory behaviors that will enhance the Bernoulli's effect to restore the glottic wave. Hyperfunctional voice disorder is a non-physiological increase in tone of the vocal folds on phonation.
\end{abstract}

Aim of the work: Is to evaluate the effects of the AM of voice therapy on acoustic measurements, in patients with hyperfunctional dysphonia.

Patients and methods: This study was applied on 25 patients with age ranging from 20 - 60 years, from January 2018 to December 2020, diagnosed with Hyperfunctional dysphonia based on objective and clinical measures, recruited from the outpatient Phoniatric clinic at Ain Shams University Hospitals, underwent acoustic analysis (CSL) pre and post therapy.

Results: Comparing pre and post therapy results, there was no statistically significant difference in fundamental frequency while there was statistically significant difference (reduction) in Jitter \%, Shimmer\% and Noise to harmonic ratio.

Conclusion: The present study showed that the AM of voice therapy improved acoustic measurements suggestive of noise to the vocal production and positive effects on the closure of the vocal folds, therefore Smith Accent method of voice therapy is an effective voice line of rehabilitation in hyperfunctional dysphonia.

Keywords: Voice therapy, Acoustic analysis, Smith Accent Method, Hyper functional dysphonia.

\section{INTRODUCTION:}

Voice disorders are characterized by abnormalities in pitch, loudness, and/or quality of the voice that can limit the effectiveness of oral communication and cannot fulfill the speaker's social and occupational requirements. ${ }^{(1)}$

Dysphonia is a general term for voice disorders representing the main symptom of laryngeal lesions; it seriously affects the life quality of patients and interpersonal communication. It is known that the primary factor for dysphonia is imperfect closure of the glottis which is easy to diagnose by laryngoscopy. Also, vocal fold stiffness or alteration of its layered structure is another factor responsible for dysphonia. ${ }^{(2)}$

Voice disorders can be classified into 
organic voice disorders, non- organic (functional) voice disorders, Minimal Associated Pathological Lesions (MAPLs) and Accompaniments of neuro-psychiatric ailments. $^{(3)}$

Non-organic voice disorders account for at least $40 \%$ of the cases of dysphonia referred to multidisciplinary voice clinics. They occur predominantly in women. Some authors refer to non-organic dysphonia as muscle tension dysphonia (MTD) or hyper functional dysphonia. ${ }^{(4)}$

In hyperfunctional dysphonia, the patient is using excessive force to produce the necessary breath support for the act of phonation. The patient is straining, there is increased subglottic pressure. Such as excessive motor power (tension) reflects also on the force of adduction and approximation of the vocal folds, and may be the ventricular bands also, while phonating. The voice is strained and leaky and may be of a relatively lower pitch. ${ }^{(5)}$

The management of voice disorders, in general, may follow one of four avenues: phonosurgery, pharmacotherapy, technical devices or behavior modification voice therapy. Hyperfunctional voice disorders are mainly treated with behavior modification voice therapy. ${ }^{(5)}$ Conservative treatments (behavior modification voice therapy) are mainly based on voice hygienic advice and voice therapy. ${ }^{(3)}$

The purpose of voice therapy is not only to provide therapeutic techniques to cure a voice disorder, but to also encourage beneficial voice habits to ensure a healthy quality of voice. In order to completely benefit from this process of voice therapy to prevent voice disorders in the future, it is crucial that an individual practices the therapeutic techniques that voice therapy advises regularly. ${ }^{(6)}$

In the domain of voice disorders, the AM of voice therapy is therapeutically indicated as a mainstream line of therapy or as a complementary one. The main applications in the former case are: (1) nonorganic (functional) habitual voice disorders in adults and children; (2) non-organic psychogenic voice disorders; (3) selected types of minimal associated pathological lesions such as vocal nodules and contact granuloma; (4) selected types of organic voice disorders such as motor disorders of the vocal folds (paralytic dysphonia).

The main application of the AM of voice therapy as a complementary line of treatment is in association with pharmacotherapy or phonosurgery. ${ }^{(7)}$

This method trains patients to produce easy voicing, abdominal breathing movements (by alternating contraction and release) and open throat postures. These productions are achieved by using rhythmic vocalizations of consonant sounds (called accents) in combination with body movements (e.g., swaying arms or rocking back and forth) while stressing respiratory support for each accent. ${ }^{(5)}$

The main goal of the AM is to achieve better control of speech and voice production. The exact mechanism for readjusting laryngeal vocal physiology is not yet clearly understood. It is believed, however, that this task is accomplished by the enhancement of the Bernoulli effect at the glottis through better pulmonary support. The latter is effected by accentuated rhythmic expiratory pulses. The psychomotor responses to the improved expiratory pulmonic pulse (improved breath support) are believed to be the result of restoration of the symmetry of the vibrator (glottis), reduction of glottic waste and reduction of excessive glottal muscular effort; hence, better voluntary control. ${ }^{(3)}$

Acoustic analysis of voice is a noninvasive method for analysis of the objective voice quality parameters in various experimental as well as clinical settings: fundamental frequency (Fo), jitter, shimmer 
and noise-to-harmonics ratio (NHR). ${ }^{(8)}$

The major clinical applications of acoustic analysis tend to fall into three broad categories: screening, diagnostic support and evaluation of the relative effectiveness of different treatment approaches, and also in assessment of progress throughout the course of treatment. ${ }^{(9)}$

\section{AIM OF THE WORK:}

This study aims to analyze the acoustic measurements after AM of voice therapy in hyperfunctional dysphonia patients in order to generalize these results in future studies.

\section{PARTICIPANTS AND METHODS:}

\section{Patients:}

25 Hyperfunctional dysphonia patients, recruited from the outpatient Phoniatric clinic at Ain Shams University Hospitals from January 2018 to December 2020, were included in this study.

\section{Selection criteria:}

\section{Inclusion criteria:}

- $\quad$ Affected habitual voice in the Auditory Perceptual Assessment (APA), with diagnosis of hyperfunctional dysphonia.

- Age range (20 years to 60 years).

\section{Exclusion criteria:}

- Other dysphonia due non-organic voice disorders.

- $\quad$ Previous micro-laryngeal surgery (MLS) for any lesion in the vocal folds.

Methods: All patients underwent the following steps:

\section{Patient interview:}

Personal Data: name, age and gender, education, occupation, residence, marital status and number of children/Complaint and analysis of symptoms: Duration - onset
- course/Impact of complaint on the subject: Patient's self-rating of dysphonia severity, effect on daily life, listener's reaction/Phonasthenic symptoms (throat dryness, throat tenderness, frequent throat clearing or difficulty in swallowing sticky throat mucous)/Symptoms related to the larynx and to voice: breathing and airway patency, chewing and swallowing, digestion and hearing/Past history of repeated upper respiratory tract infection, laryngeal, neck and other surgeries/Habits and situations damaging to voice: smoking, excessive faulty use of voice and harsh working environmental factors/Associated health problems: chronic cough, asthma, autoimmune diseases and allergies.

Auditory perceptual assessment with tabulation of the results following modified GRBAS scale, a 4-degree scale, as follows: Overall grade of dysphonia: "(0) Normal, (1) slight, (2) moderate, (3) severe"/Character (quality): "strained- leaky- breathy- rough (irregular)" /Pitch: "overall increasedecrease- diplophonia" /Loudness: "excessively loud- excessively softfluctuating- average".

Acoustic analysis was done for the voice of every patient (pre therapy and post therapy) in a sound treated room by recording sustained /a/ vowel at a comfortable level of loudness with a microphone at a distance of about $15 \mathrm{~cm}$ from the mouth. The recording was then subjected to acoustic measurements using a computerized multidimensional voice program and analysis of 4 parameters (fundamental frequency, jitter\%, shimmer\% and Noise to harmonic ratio).

\section{Statistical Analysis:}

\section{i. Descriptive statistics:}

1. Mean \pm SD (standard deviation) and Range for parametric numerical data. Median and Interquartile range (IQR) for non-parametric numerical data.

2. Frequency/Number and Percentage of 
non-numerical data.

\section{ii. Analytical statistics: Paired t-test was} used to assess the statistical significance

\section{RESULTS}

The distribution of age and gender of of the difference between the two means measured twice for the same study group.

the participated hyperfunctional dysphonia patients is showed in Table 1 and Table 2.

I. Age and Gender: "Tables 1 and 2"

Table 1: The distribution of the sex among hyperfunctional dysphonia patients.

\begin{tabular}{|c|c|c|c|}
\hline \multicolumn{2}{|c|}{} & Number & $\%$ \\
\hline \multirow{2}{*}{ Sex } & Male & 13 & $52 \%$ \\
\cline { 2 - 4 } & Female & 12 & $48 \%$ \\
\hline
\end{tabular}

Table 2: The distribution of the gender among hyperfunctional dysphonia patients.

\begin{tabular}{|c|c|c|}
\hline & Mean \pm SD & Range \\
\hline $\begin{array}{c}\text { Age } \\
\text { (years) }\end{array}$ & $37.12 \pm 10.47$ & $21-59$ \\
\hline
\end{tabular}

II. Aoustic Analysis "Fundamental frequency, Jitter \%, Shimmer \% and Noise to harmonic Ratio" are shown in (Table 3 \&Diagram 1), (Table 4 \&

Diagram 2), (Table 5 \& Diagram 3) and (Table 6 \& Diagram 4) respectively.

Table 3: Comparison between Fundamental frequency pre and post therapy.

\begin{tabular}{|c|c|c|c|c|c|c|c|}
\hline fundamental frequency $(\mathrm{Hz})$ & \multicolumn{2}{|c|}{ Pre-therapy } & \multicolumn{2}{|c|}{ Post-therapy } & \multicolumn{3}{|c|}{ Paired test } \\
\cline { 2 - 8 } & Mean & SD & Mean & SD & T & $\begin{array}{c}\mathrm{p} \\
\text { value }\end{array}$ & significance \\
\hline $\mathrm{n}=25$ & & & & & & & \\
\hline
\end{tabular}

$n=$ number (sample size $), N S=$ Non-significant

Comparing pre and post therapy results, difference in fundamental frequency. there was no statistically significant

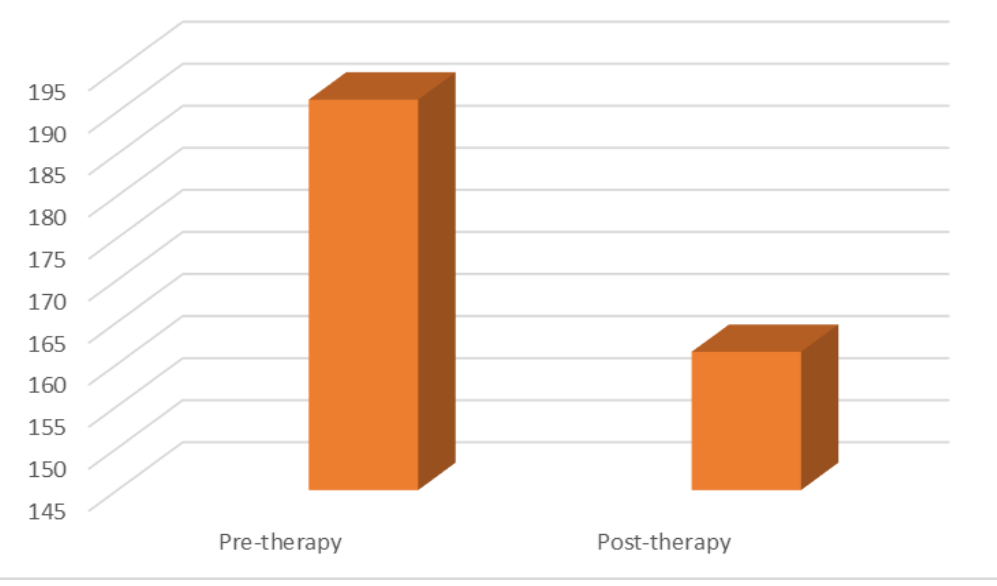

Diagram (1): Comparison of pre and post therapy fundamental frequency. 
Efficacy of The Accent Method of Voice Therapy on Acoustic Measurements, In Hyperfunctional...

Table 4: Comparison between Jitter \% pre and post therapy.

\begin{tabular}{|c|c|c|c|c|c|c|c|}
\hline \multirow{2}{*}{ Jitter \% } & \multicolumn{3}{|c|}{ Pre } & \multicolumn{3}{|c|}{ Post } & Paired test \\
\cline { 2 - 8 } & Mean & SD & Mean & SD & T & $\begin{array}{c}\mathrm{p} \\
\text { value }\end{array}$ & significance \\
\hline $\mathrm{n}=25$ & 3.11 & 2.41 & 1.02 & 0.60 & 4.35 & $<0.001$ & $\mathrm{~S}$ \\
\hline
\end{tabular}

$n=$ number (sample size), $S=$ significant

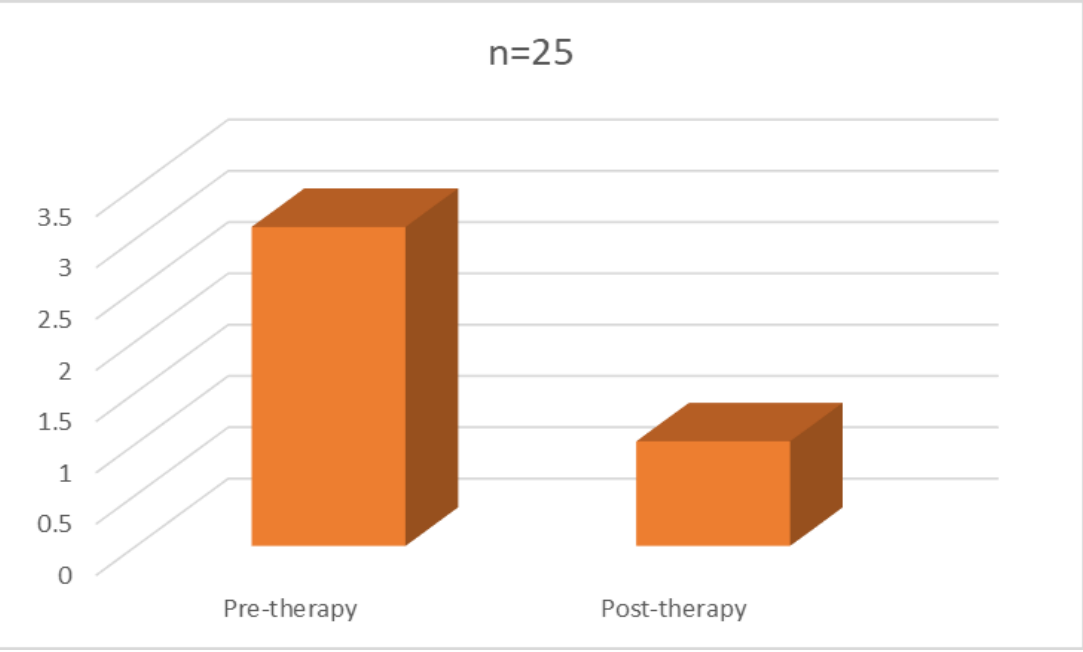

Diagram (2): Comparison of pre and post therapy Jitter \%

Comparing pre and post therapy results, in Jitter $\%$. there was statistically significant difference

Table 5: Comparison between Shimmer \% pre and post therapy.

\begin{tabular}{|c|c|c|c|c|c|c|c|}
\hline \multirow{2}{*}{ Shimmer \% } & \multicolumn{3}{|l|}{ Pre-therapy } & \multicolumn{3}{l|}{ Post-therapy } & \multicolumn{3}{l|}{ Paired test } \\
\cline { 2 - 8 } & Mean & SD & Mean & SD & T & p value & significance \\
\hline $\mathrm{n}=25$ & 4.92 & 2.78 & 2.00 & 0.84 & 5.46 & $<0.001$ & $\mathrm{~S}$ \\
\hline
\end{tabular}

$n=$ number (sample size), $S=$ significant

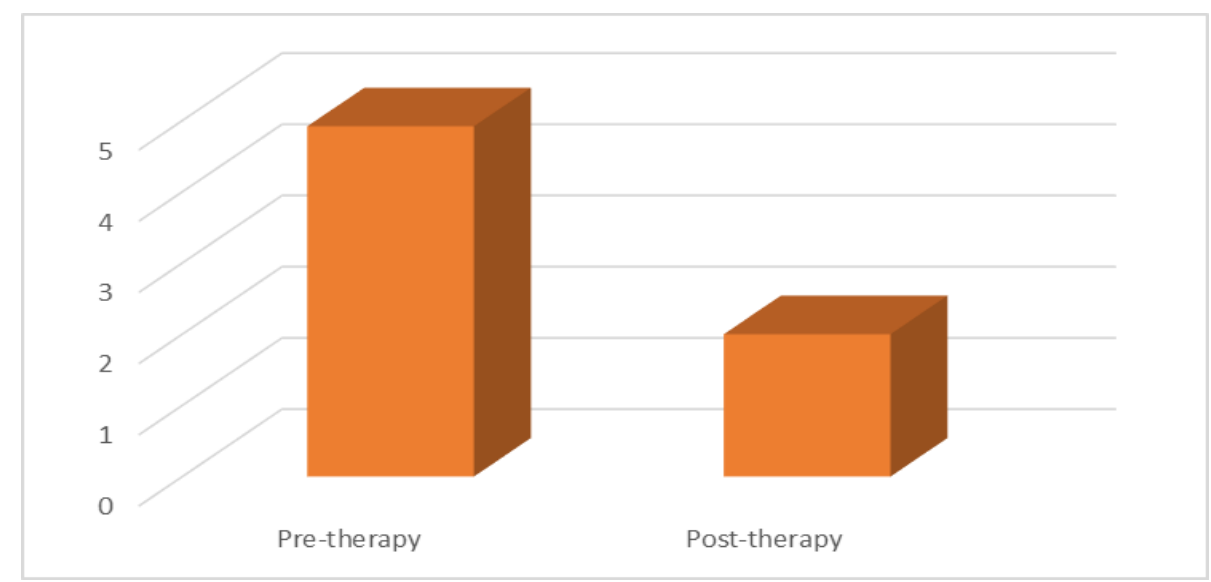

Diagram (3): Comparison of pre and post therapy Shimmer \%.

Comparing pre and post therapy results, in Shimmer \%. there was statistically significant difference 
Table 6: Noise to Harmonic Ratio pre and post therapy.

\begin{tabular}{|c|c|c|c|c|c|c|c|}
\hline Noise to harmonic ratio & \multicolumn{2}{|c|}{ Pre-therapy } & \multicolumn{2}{|c|}{ Post-therapy } & \multicolumn{4}{c|}{ Paired t test } \\
\cline { 2 - 8 } & Mean & SD & Mean & SD & $\mathrm{t}$ & $\mathrm{p}$ value & significance \\
\hline $\mathrm{n}=25$ & 0.23 & 0.16 & 0.12 & 0.02 & 3.66 & 0.001 & $\mathrm{~S}$ \\
\hline
\end{tabular}

$n=$ number (sample size $), S=$ significant

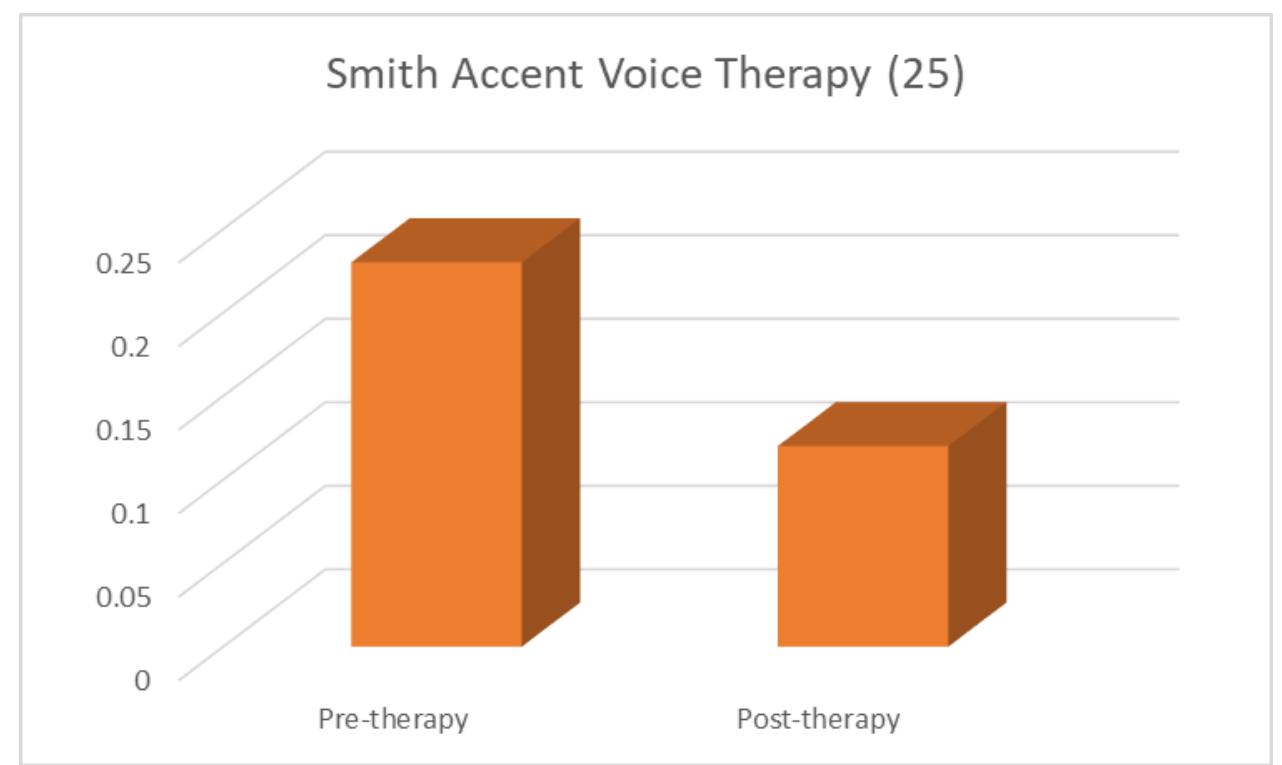

Diagram (4): Comparison of pre and post therapy Noise to Harmonic Ratio. Comparing pre and post therapy results, there was statistically significant difference in Noise to Harmonic Ratio.

\section{DISCUSSION:}

The general goal of voice therapy is to rehabilitate the patient's voice to a level of function that enables the patient to fulfill his or her daily voice and/or speech communication needs ${ }^{(\mathbf{1 2}) \text {. }}$

In this study, 25 hyperfunctional dysphonic patients underwent AM of voice therapy. Acoustic analysis was done before and 1 month after the completion of the therapy.

On perceptual analysis of patients with hyperfunctional dysphonia, their voice

This is in accordance to Fex et al. $(1994)^{(14)}$ who conducted a study on ten patients diagnosed as functional voice disorders and recieved AM of voice therapy. The vocal folds were normal except in three females who had moderate bilateral nodules. The voices were analyzed acoustically before and after treatment. Results showed samples were categorized as 'moderate' 'breathy/strained. Increase in jitter $(100 \%)$ and shimmer $(66.6 \%)$ was seen. Noise-toHarmonic Ratio includes contributions from both perturbations of amplitude and frequency. Thus, it provides a compilation of jitter and shimmer. The ratio is $<1$ in normal voice ${ }^{(\mathbf{1 3})}$.

In this study, there was a significant improvement in fundamental frequency and significant reduction in Jitter $\%$ and Shimmer $\%$ on comparing pre and post therapy results.

significant reduction in pitch perturbation quotient "jitter" and amplitude perturbation quotient "shimmer", normalized noise energy, and fundamental frequency showed significant improvement.

Bassiouny(1998) ${ }^{(7)}$ and Bassiouny et al. $(2019)^{(15)}$ conducted a study in order to evaluate the efficacy of the AM of voice 
therapy. The study included 100 patients: 50 adult patients of both sexes received "accent exercises" of voice therapy 1 week after microlaryngeal phonosurgery which was the study group (G1) and other 50 adult patients of both sexes as a control group (G2) received only voice hygienic advices. Results regarding acoustic analysis, showed that there was evidence for the difference in improvement of all parameters in between the groups (G1 and G2) in favor of (G1) because of the role of the rhythmic accentuated accent exercises on sound pressure level (SPL) to increase airflow rate and to produce variable degrees of increase in fundamental frequency (Fo) and decrease in Jitter \%, Shimmer $\%$ and Noise to Harmonic Ratio (NHR).

Khidr (2003) ${ }^{(\mathbf{1 6})}$ performed study received AM of voice therapy, resulted in, significant improvement in all of the acoustic parameters.

\section{Conclusion \& Recommendations:}

AM of voice therapy is effective in improving the voice quality of hyperfuctional dysphonic patients.

Further work needs to be done on a large-scale to compare the effectiveness of AM of voice therapy to other types of voice therapy (e.g: Semi- ocludded Vocal Tract Exercises "SOVTE", relaxation methods, vocal function exersices and resonant voice therapy in dysphonic patients).

\section{REFERENCES}

1. Simberg, S., Sala, E. and Tuomainen, J. (2006): The Effectiveness of Group Therapy for Students With Mild Voice Disorders: A Controlled Clinical Trial. Voice J.; 20(1):97-109.

2. American Speech-Language Hearing Association (ASHA) (2016): Knowledge and skills for speech-language pathologists with respect to Behavioral readjustment therapy (the Accent Method). Retrieved from www.asha.org/policy.
3. Kotby, M.N., El-Sady, S.R., Bassiouny, S.E., Abou Rass, Y.A. and Hegazi, M.A., (1991): Efficacy of the Accent Method of voice therapy. Journal of voice, 5:316.

4. Werning, J., McAllister, L. and Antush, K. (2007): Functional voice disorders. Available from: http://emedicine.medscape. com/article/865191- overview.

5. Kotby, M. N., El-Assal,N., Gamal, N., Rifaie, N., and Dawood,A. (2005): Efficacy of the accent method of voice therapy in treatment of vocal fold paralysis. AinShams Med. J. 2005; 56 (4,5,6): 663-671.

6. Phillips, M.C. (2012): Voice Therapy: A Safe and Effective Alternative to Voice Surgery. J voice; 4(8):1-2.

7. Bassiouny, S. (1998): Efficacy of the accent method of voice therapy. Folia Phoniatrica et Logopedica, 50: 146-164.

8. Amir, O., Wolf, M. and Amir, N. (2009): A clinical comparison between two acoustic analysis softwares: MDVP and Praat, Biomedical Signal Processing and Control; 4(3): 202-205, DOI: 10.1016/j.bspc.2008. 11.002 .

9. Laver, J., Hiller, S. and Beck, J.M. (1992): Acoustic waveform perturbations and voice disorders. Journal of Voice, 6, 115-126.

10. Kotby (1995): The accent method of voice therapy. San Diego: Singular Publishing Group

11. Kotby M.N., Baraka, M., Abou El Ella, M.Y., Elsady, S., Elshoubary,A. and Refaie, N. (2016): Introduction to vocology, clinical vocology; 2 (1):29-78

12. Echternach, M., Guzman, M., Köberlein M., Gellrich, D., Laukkanen, A.,Burk, F., Döllinger, M., Richter, B. and Kainz, M. (2020): Duration of biodynamic changes associated with water resistance therapy, Logopedics Phoniatrics Vocology, DOI: 10.1080/14015439. 2020. 1785000

13. Kavita, S. and Tanvi, S. (2018): Dysphonia and its Correlation with Acoustic Voice Parameters, International Journal of Phonosurgery and Laryngology;8 (1):6-12

14. Fex, B., Fex, S., Shiromoto, O. and Hirano, 


\section{Nashwa Mahmoud Othman, et al.,}

M. (1994): Acoustic analysis of functional dysphonia: before and after voice therapy (accent method). J Voice; 8(2):163-7, doi: 10.1016/s0892-1997(05) 80308.

15. Bassiouny, S., El-Shobary, A., Hafez, N., Shawky, A. and Mousa, A. (2019): Evaluation of the role of the accent method of voice therapy after microlaryngeal phonosurgery. Ain Shams Medical Journal; (70): DOI: 10.21608/asmj.2019.101266

16. Khidr, A. (2003): Effects of the "Smith Accent Technique" of voice therapy on the laryngeal function and voice quality of patient with unilateral vocal fold paralysis. J Voice, 1240(8): 1235-1241. DOI: 10.1016/S0531-5131 (03) 00836-7.

\section{كفاءة الطريقة الإيقاعية لسميث على القياسات الصوتية لمرضى البحة الصوتية فوق الوظيفية

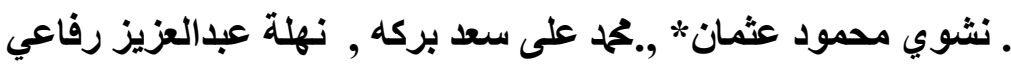

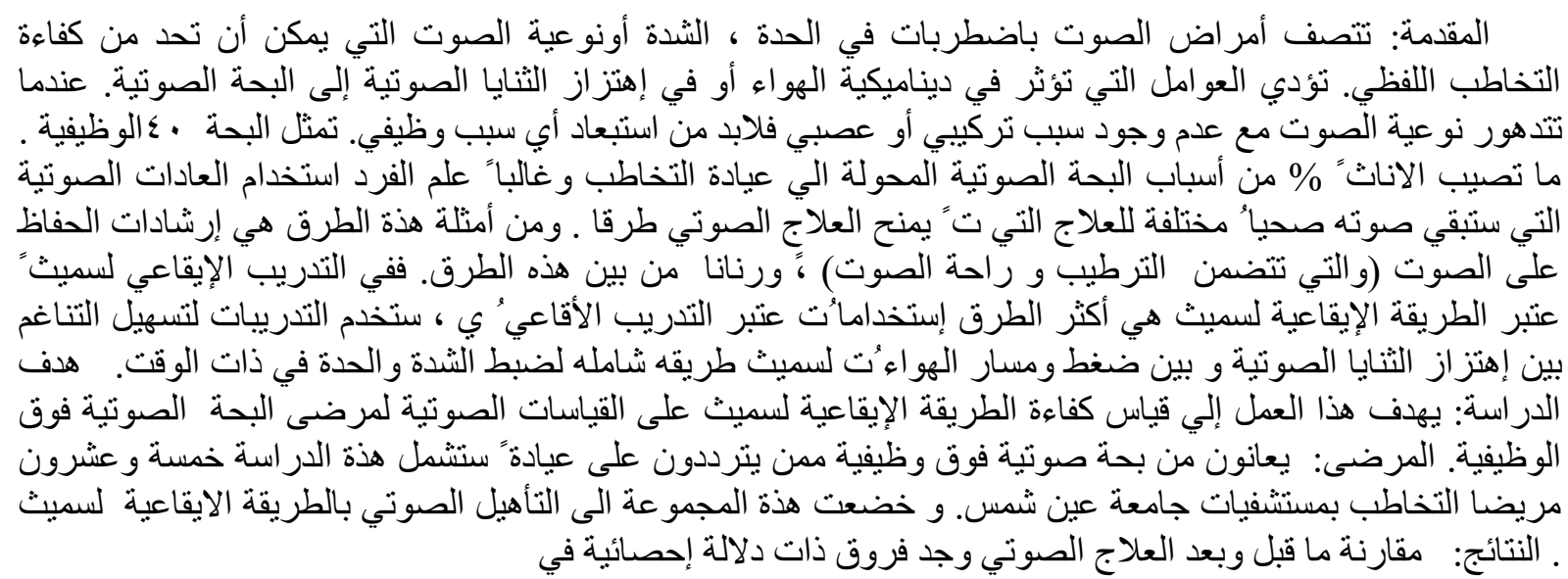

Jitter\%, Schimmer\% and Noise to harmonic ratio

الخلاصة :ان العلاج الصوتي بالطريقة الإيقاعية لسميث فعال في تحسين اضطر ابات الصوت لاى المرضى الذين بعانون من البحة الصوتية فوق الوظيفية. 